\title{
Techniques for precise energy calibration of particle pixel detectors
}

M. Kroupa, T. Campbell-Ricketts, A. Bahadori, and A. Empl

Citation: Review of Scientific Instruments 88, 033301 (2017); doi: 10.1063/1.4978281

View online: http://dx.doi.org/10.1063/1.4978281

View Table of Contents: http://aip.scitation.org/toc/rsi/88/3

Published by the American Institute of Physics

\section{Articles you may be interested in}

Space-resolved visible spectroscopy for two-dimensional measurement of hydrogen and impurity emission spectra and of plasma flow in the edge stochastic layer of LHD

Review of Scientific Instruments 88, 033501033501 (2017); 10.1063/1.4976963

Suppression of inter-device variation for component analysis of turbid liquids based on spatially resolved diffuse reflectance spectroscopy

Review of Scientific Instruments 88, 033104033104 (2017); 10.1063/1.4977788

Fast, precise, and widely tunable frequency control of an optical parametric oscillator referenced to a frequency comb

Review of Scientific Instruments 88, 033101033101 (2017); 10.1063/1.4977049

Development and experimental study of oil-free capacitor module for plasma focus device

Review of Scientific Instruments 88, 033502033502 (2017); 10.1063/1.4977218 


\title{
Techniques for precise energy calibration of particle pixel detectors
}

\author{
M. Kroupa, ${ }^{1,2, a)}$ T. Campbell-Ricketts, ${ }^{3}$ A. Bahadori, ${ }^{4}$ and A. Empl ${ }^{3}$ \\ ${ }^{1}$ Leidos, Houston, Texas 77258-8487, USA \\ ${ }^{2}$ Space Radiation Analysis Group, NASA Johnson Space Center, Houston, Texas 77058, USA \\ ${ }^{3}$ University of Houston, Houston, Texas 77004, USA \\ ${ }^{4}$ Department of Mechanical and Nuclear Engineering, Kansas State University, Manhattan, Kansas 66506, USA
}

(Received 3 November 2016; accepted 23 February 2017; published online 13 March 2017)

\begin{abstract}
We demonstrate techniques to improve the accuracy of the energy calibration of Timepix pixel detectors, used for the measurement of energetic particles. The typical signal from such particles spreads among many pixels due to charge sharing effects. As a consequence, the deposited energy in each pixel cannot be reconstructed unless the detector is calibrated, limiting the usability of such signals for calibration. To avoid this shortcoming, we calibrate using low energy X-rays. However, charge sharing effects still occur, resulting in part of the energy being deposited in adjacent pixels and possibly lost. This systematic error in the calibration process results in an error of about $5 \%$ in the energy measurements of calibrated devices. We use FLUKA simulations to assess the magnitude of charge sharing effects, allowing a corrected energy calibration to be performed on several Timepix pixel detectors and resulting in substantial improvement in energy deposition measurements. Next, we address shortcomings in calibration associated with the huge range (from kiloelectron-volts to megaelectron-volts) of energy deposited per pixel which result in a nonlinear energy response over the full range. We introduce a new method to characterize the non-linear response of the Timepix detectors at high input energies. We demonstrate improvement using a broad range of particle types and energies, showing that the new method reduces the energy measurement errors, in some cases by more than $90 \%$. Published by
\end{abstract} AIP Publishing. [http://dx.doi.org/10.1063/1.4978281]

\section{INTRODUCTION: DOSIMETRY WITH PIXEL DETECTORS}

Improvements in microelectronics allow for ever more complex pixel architectures inside modern semiconductor radiation detectors. Dedicated front-end electronics can now be included in each pixel, allowing the capability to measure the deposited energy, and occasionally timing information, independently in each element. For these reasons, pixel detectors are broadly used in high energy physics and are becoming popular in space dosimetry. ${ }^{1-4}$ In dosimetric applications, they not only provide the measured flux but allow for precise $\mathrm{dE} / \mathrm{dx}$ measurements, ${ }^{5-7}$ and hence species and velocity analysis. New radiation detectors for future exploration missions developed by NASA are based on the Timepix pixel detectors, ${ }^{8,9}$ a product of the Medipix 2 collaboration.

Timepix is a hybrid pixel detector, with a matrix of $256 \times 256$ pixels, each being $55 \times 55 \mu \mathrm{m}^{2}$ square outline. Ana$\log$ front-end electronics in each pixel provide charge amplification and shaping, while a digital front end allows for operation in one of three modes: counting mode, Time over Threshold (TOT) mode, or Time of Arrival (TOA) mode. The TOT mode is analogous to a multi-channel analyzer, allowing the measurement of charge collected in the pixel. The signal resulting from an ionizing radiation event is shaped in the Timepix front end to an almost triangular voltage pulse. Using our preferred setting of $\mathrm{I}_{\text {krum }}=2$ ( $\mathrm{I}_{\text {krum }}$ is the Krummenacher current, ${ }^{10}$ which determines the pulse discharge speed), the typical

\footnotetext{
a) Author to whom correspondence should be addressed. Electronic mail: martin.kroupa@nasa.gov
}

length of this pulse is from microseconds up to hundreds of microseconds depending on the input signal. In the TOT mode, the number of clock cycles (we use a $10 \mathrm{MHz}$ clock) over which the voltage pulse remains above an adjustable threshold is used to calculate the energy content of the event. This threshold applies to all pixels; it is set above the detector noise distribution and thus allows the elimination of all dark counts.

Accurately calibrating the Timepix for particle detection is highly challenging and typically a combination of gamma sources and fluorescent foils has been used, as described by Jakubek. ${ }^{11}$ Settings suitable for many applications are discussed in Kroupa et al. ${ }^{12}$ We found that $\mathrm{I}_{\mathrm{krum}}$ set to 2 gives us best resolution while still allowing the energy measurement of heavy ions, where for lower $\mathrm{I}_{\text {krum }}$ one could reach the counter limit (11 810 clocks). Due to internal differences between pixels, the calibration must be performed on a pixel-by-pixel basis.

\section{CORRECTED CALIBRATION}

To calibrate devices we start with Jakubek's procedure utilizing laboratory gamma sources. Calibration data are collected using $5 \mathrm{mCi}{ }^{241} \mathrm{Am}$ and $1 \mathrm{mCi}{ }^{55} \mathrm{Fe}$ radioactive sources. The sources are placed in a holder roughly $1 \mathrm{~cm}$ above the sensor. Thin tin foil is positioned in between the americium source and sensor, providing an extra fluorescence line. The three points used for calibration are $5.99 \mathrm{keV}(\mathrm{Fe}), 25.27 \mathrm{keV}(\mathrm{Sn})$, and $59.54 \mathrm{keV}(\mathrm{Am})$.

Charge-sharing effects between pixels, ${ }^{13-16}$ however, lead to a systematic error in the calibration process, which becomes 
amplified with high-energy deposition events. Due to the finite thickness of the device (we use silicon sensors of 300 and $500 \mu \mathrm{m}$ thicknesses and threshold of approximately $5 \mathrm{keV}$ ), the energy deposited at a single point produces a signal that can spread in several adjacent pixels. An X-ray photon interacting with a silicon sensor, for example, creates a photo-generated charge cloud, which is then separated by the detector bias voltage and collected at the electrodes. Diffusion, Coulombic repulsion, and possibly other effects, collectively referred to as charge sharing, lead to the charge cloud spreading laterally during the vertical transport to the collection electrodes and can cause a point-like energy deposition to produce signals in multiple pixels. The total deposited energy is then proportional to the sum of the signals from all those pixels. However, if the signal deposited in the pixel is lower than threshold, the information is lost. We call the set of active pixels associated with one incident particle a cluster, the total energy deposited by a particle is referred to as the cluster volume, and the cluster height is defined as the value of the energy deposited in the pixel with the largest signal within the cluster.

When calibrating pixel detectors, the preferred procedure is to accept only those events where the deposited energy is registered in just a single pixel. This is because, prior to calibration, due to pixel differences, there is no easy way to determine the proportions of the total incoming energy received in each pixel in a multi-pixel event. Single pixel events, however, have a high probability of registering less than the total deposited energy, as neighboring pixels may receive a small amount of charge that falls below the detection threshold. This can be seen in Figure 1, which shows the dependence of the spectra measured over a $59.54 \mathrm{keV}^{241} \mathrm{Am}$ line, on the size of the pixel clusters produced. Pixel-by-pixel calibration was performed, using the standard single-pixel-event procedure, and so the peak of the single-pixel spectrum in Figure 1 is very close to the true value. For clusters with 4 pixels, however, the mean of total reconstructed energy is $66.5 \mathrm{keV}$. As this value is higher than the total energy of the interacting photon, we could assume that there is a systematic error in the calibration

\section{$59.54 \mathrm{keV}$ line different cluster size}

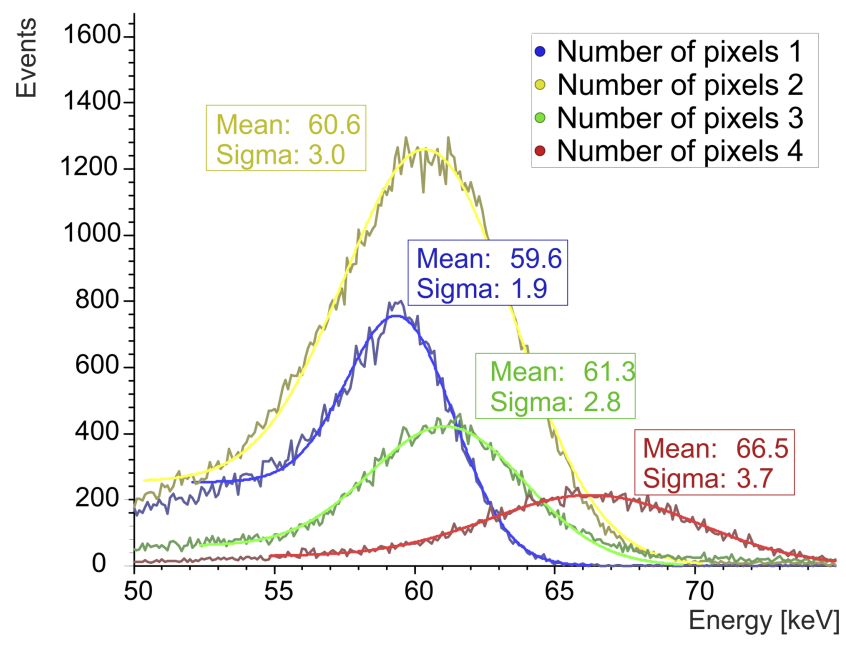

FIG. 1. Example of overestimation of calibration using a ${ }^{241} \mathrm{Am}$ gamma source. process caused by the fact that the energies recorded in the single-pixel calibration events were substantially lower than the energy of the incident photons.

The energy of the ${ }^{241} \mathrm{Am}$ line is a practical maximum one can use for calibration. As stated earlier, one must know the deposited energy in the pixel in order to use the signal for energy calibration, requiring the signal to be deposited entirely within one pixel. If more pixels are affected, the signal cannot be used because the energy distribution among the pixels cannot be determined from measurement. For a Timepix with a $500 \mu \mathrm{m}$ sensor, less than one third of the events from ${ }^{241} \mathrm{Am}$ gamma source deposit signal into only one pixel. For higher energies, it would be difficult to obtain enough single pixel events for calibration in a reasonable timeframe.

\section{A. FLUKA simulation}

To assess the effect of charge sharing on the calibration procedure, a FLUKA simulation study of the physical processes leading to energy deposition in the Timepix sensor was performed. FLUKA is a general purpose tool for calculations of particle transport and interactions with matter, covering an extended range of applications. ${ }^{17,18}$ The version of FLUKA used for the present study is FLUKA2011.2 from October 2014. A detailed description of the Timepix detector, the surrounding holder and the radioactive ${ }^{241} \mathrm{Am}$ source together with the tin foil, was implemented in the simulation. Each detector pixel was subdivided into $11 \times 11 \times 100$ cubic subvolumes with an edge of $5 \mu \mathrm{m}$ for the purpose of precisely recording the energy deposition of particles traversing the silicon sensor volume. A simple model was developed in order to predict the charge carriers created in the sensor and their collection based on the energy deposited per sub-volume. This post-processing step addresses in a quasi-static approach both the diffusion of the charge carriers in the sensor volume during their vertical drift and induction of a signal on the collection plane.

Special attention was given to the electronic noise. An empirical description of the per-pixel noise was derived making use of the experimentally determined threshold distribution. A few free parameters in the model were determined by comparing predicted to measured spectra. The simulation estimates the amount of charge lost, below threshold, in neighboring pixels, when photons of the energies corresponding to the calibration sources are detected in single-pixel events, giving the actual amount of energy corresponding to each measured TOT. This correction is performed globally, rather than on a pixel-by-pixel basis.

The estimate of the deposited energy in the pixel from FLUKA for all three calibration lines is used as the input for calibration. New calibration is evaluated using data from stopping particles with well-known energy.

\section{B. Verification}

We verified the corrected calibration procedure using data obtained at the Tandem Van de Graaff facility at Brookhaven National Laboratory, using stopping protons of $5 \mathrm{MeV}$. It is important to say, however, that our simulation depends on 
TABLE I. Corrected calibration for 5 devices with $500 \mu \mathrm{m}$ silicon chip.

\begin{tabular}{lc}
\hline \hline $\begin{array}{l}\text { Photon energy } \\
(\mathrm{keV})\end{array}$ & $\begin{array}{c}\text { Estimated single-pixel } \\
\text { deposited energy_average of } \\
5 \text { devices }(\mathrm{keV})\end{array}$ \\
\hline 5.899 & $5.62 \pm 0.04$ \\
25.27 & $23.88 \pm 0.08$ \\
59.541 & $56.74 \pm 0.35$ \\
\hline \hline
\end{tabular}

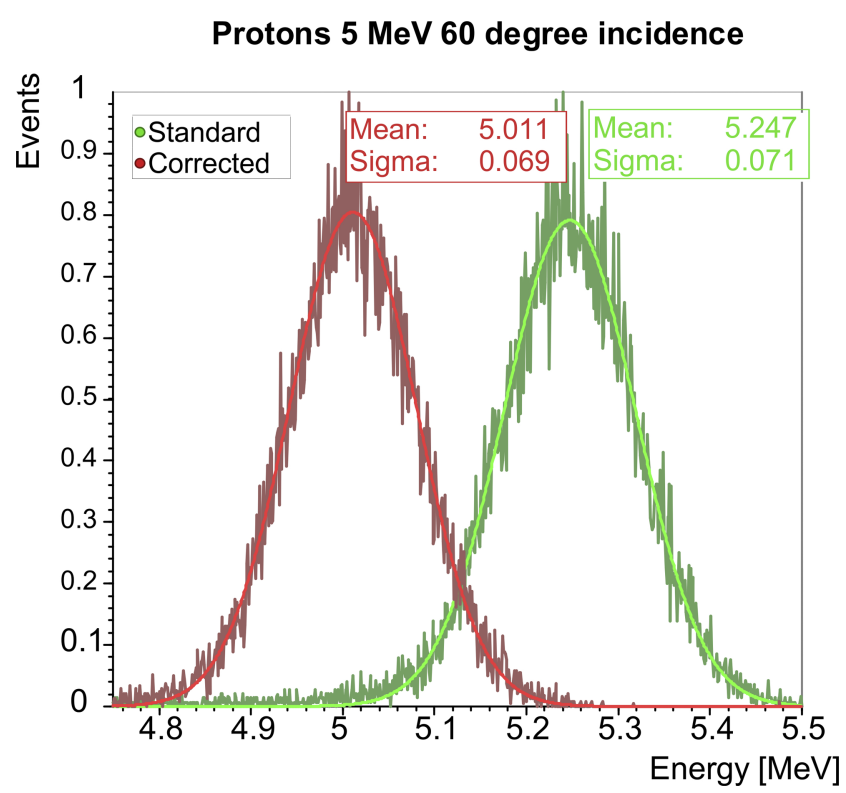

FIG. 2. Comparison of the standard ("Old coef") and corrected ("New coef") calibration for $5 \mathrm{MeV}$ stopping protons.

internal parameters of the particular device, such as noise level and gain. Consequently, because we only produced the FLUKA simulation for a single device, we made small adjustments to the FLUKA-estimated parameters, until the best match between experimental and expected spectra was obtained. The estimates of the deposited energy using statistics from five devices are shown in Table I. In our comparisons, we will call calibration using the FLUKA-corrected input spectrum "corrected," while the calibration using the uncorrected source energies will be referred to as "standard."

Figure 2 shows the improved accuracy (more than 90\% reduction of accuracy error, final accuracy better than 1\%), when corrected input spectrum is used for calibration. The precision of the measurement is not affected. The stopping protons of $5 \mathrm{MeV}$ were used to demonstrate this improvement, as those fully stop within the device and deposit all energy. Data were taken inside the vacuum chamber to eliminate energy losses in the air in order to guarantee best accuracy.

\section{ADVANCED CALIBRATION}

\section{A. Method}

Another difficulty with the Timepix that makes calibration verification complex is the so-called saturation effect, ${ }^{12}$ resulting from oscillation of the front-end preamplifier under very large signals $(>1 \mathrm{MeV} /$ pixel). This effect is caused by undershooting of the preamplifier voltage which occurs for large input charges. This undershoot is processed in the comparator as a second, false signal. As the Timepix logic sums all signals during a measurement, the result of measurement with very large signal is the sum of the real and false ones.

Investigation showed that the saturation effect is in fact very similar to the behavior of the double-linear preamplifier. The response curve exhibits a linear region (except for a very low energy knee) up to about $850 \mathrm{keV}$, which is calibrated using either standard or our corrected procedure (referred to as the regular region). Above $1 \mathrm{MeV}$ there is another linear part, but with a markedly different slope (referred to as the oscillation region). Between these two linear regions, there is a pronounced non-linearity in the response, meaning that there is no one-to-one correspondence between incoming energy and recorded TOT. This effect also means that the calibration is valid only for events where the signal is smaller than $850 \mathrm{keV} /$ pixel. To overcome this shortcoming, we developed an advanced calibration procedure.

As mentioned above, it is difficult to deliver a known signal higher than $100 \mathrm{keV}$ into the pixel. The advanced calibration procedure addresses this difficulty by collecting data using stopping protons at the Tandem Van de Graaff facility at different angles of incidence. The particle track length inside the pixel (and as a consequence also the cluster height) depends strongly on the incidence angle. The particle type and energy must be selected so that for perpendicular incidence, the response for the highest pixel in the cluster is in the oscillation region, while at shallow angles, the cluster height remains below $850 \mathrm{keV}$ at regular region. We select particle type and energy such that they stop completely inside the detector for all incident angles. The cluster volume should be the same for all angles, as we measure the total energy of the particle regardless of the angle of incidence. However, we observed that the cluster volume is higher for steep angles (closer to normal incidence), as the signal switches to the second linear part (oscillation region) and the modelled response from the original or corrected calibration is no longer applicable. In order to determine the slope of the oscillation region, we need to use events where only one pixel is collecting a large enough signal to be in the oscillation region. This pixel value corresponds then to cluster height. As the position of the oscillation region is not clear, we tested the procedure with different limits ranging from $800 \mathrm{keV}$ to $1100 \mathrm{keV}$. The method is found to be robust with respect to definition of the oscillation region and to the limit energy, and we currently use $850 \mathrm{keV}$.

The measured cluster height is then corrected by the difference between the measured cluster volume and the cluster volume from the shallow-angle measurement. The energy of the protons we used was $5 \mathrm{MeV}$ and results are shown in Figure 3. As modelling the spread of deposited charge inside the sensor is quite difficult, the particle energies suitable for this correction process are most safely determined by trial and error. The results, shown in Figure 3, give a linear correction for the oscillation region. Advanced calibration is then used after corrected calibration, if the signal in the pixel is higher than $850 \mathrm{keV}$. 


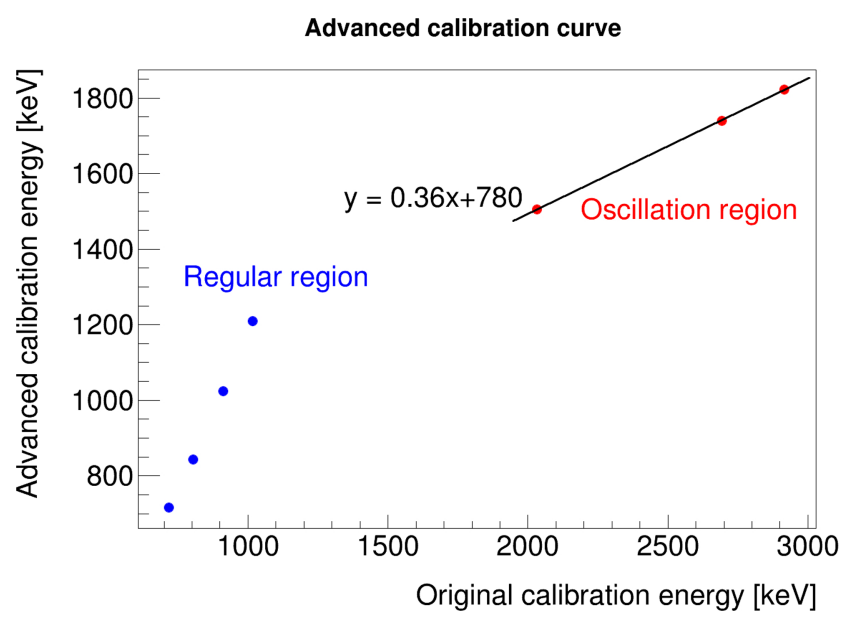

FIG. 3. Example of measured detector response function for $5 \mathrm{MeV}$ protons measured under different incident angles (going from 60 to 0 with $10^{\circ}$ step, from left to right). The blue data correspond to the regular region and red data to the oscillation region. Black is a linear fit for the oscillation region.

\section{B. Verification and testing}

We verified the new calibration in a broad range of $\mathrm{dE} / \mathrm{dx}$ from low energy protons up to high energy neon. Tables II and III show that there is improvement for both regular and oscillation regions when using our corrected and advanced calibration. For stopping particles, results are shown in Table II, for each particle type and energy, we formed a histogram of cluster volumes and used a Gaussian fit to characterize the accuracy of the cluster energy determination. For penetrating particles, we fitted the measured $\mathrm{dE} / \mathrm{dx}$ spectra, with the Landau-Vavilov distribution, using particle energy as a free parameter. Results of reconstructed particle energy are shown in Table III. The uncorrected calibration has a ten- dency to over-predict deposited energy (positive error in Table II), which corresponds to a lower estimate of particle energy when the Landau-Vavilov fit is made (negative error in Table III).

\section{SUMMARY}

We demonstrate techniques to correct two deficiencies in the energy measurements in Timepix detectors. The first effect we correct for is charge sharing, where originally a pointlike signal is spread among multiple pixels. This results in a discrepancy between the incident and measured single-pixel energies, when exposing the detector to gamma sources used for calibration. This effect is common to many types of pixel detector and becomes more serious with smaller pixel sizes. We use Monte Carlo simulations to calculate the required correction. The second effect we correct for is a "saturation" effect observed in the Timepix, when the charge collected in the pixel corresponds to more than about $850 \mathrm{keV}$. This effect results in a steeper linear response at high energy deposition. We characterize this steeper response using stopping protons producing multipixel signals, selecting only those cases where the measured energy exceeds $850 \mathrm{keV}$ in only one pixel. While this saturation effect is peculiar to the Timepix, the resulting regions of different linear response might occur in other ways, when pixel detector readout circuitry is designed to accommodate a very large range of energy depositions (from $\mathrm{keV}$ to $\mathrm{MeV}$ range). We thus anticipate that our methods might be useful for a substantial class of pixel radiation detectors. We verified our new calibration with both penetrating and stopping particles from protons to neon showing improvement in energy measurement accuracy and lowering the error in some cases by $90 \%$.

TABLE II. Absolute verification of the advanced calibration using stopping particles.

\begin{tabular}{|c|c|c|c|c|c|c|}
\hline Primary & $\begin{array}{c}\text { True } \\
\text { energy } \\
(\mathrm{MeV} / \mathrm{A})\end{array}$ & $\begin{array}{c}\text { Uncorrected } \\
\text { fit } \\
(\mathrm{MeV} / \mathrm{A})\end{array}$ & $\begin{array}{c}\text { Corrected } \\
\text { fit } \\
(\mathrm{MeV} / \mathrm{A})\end{array}$ & $\begin{array}{c}\text { Uncorrected } \\
\text { cluster } \\
\text { height }(\mathrm{keV})\end{array}$ & $\begin{array}{c}\text { Error } \\
\text { uncorrected } \\
\%\end{array}$ & $\begin{array}{c}\text { Error } \\
\text { corrected } \\
\%\end{array}$ \\
\hline $\mathrm{H}$ & 5 & 5.2 & 5.0 & 2272 & 4.0 & 0.0 \\
\hline $\mathrm{Li}$ & 10 & 12 & 9.7 & 2683 & 20.0 & -3.0 \\
\hline $\mathrm{Li}$ & 12 & 15.6 & 11.8 & 3125 & 30.0 & -1.7 \\
\hline
\end{tabular}

TABLE III. Verification of the advanced calibration for penetrating particles.

\begin{tabular}{lcccccc}
\hline \hline & $\begin{array}{c}\text { True } \\
\text { energy } \\
\text { Primary }\end{array}$ & $\begin{array}{c}\text { Uncorrected } \\
\text { (MeV/A) }\end{array}$ & $\begin{array}{c}\text { Corrected } \\
\text { (MeV/A) }\end{array}$ & $\begin{array}{c}\text { Uncorrected } \\
\text { fit } \\
\text { cluster }\end{array}$ & $\begin{array}{c}\text { Error } \\
\text { uncorrected } \\
\%\end{array}$ & $\begin{array}{c}\text { Error } \\
\text { corrected } \\
\%\end{array}$ \\
\hline $\mathrm{H}$ & 480 & 424.3 & 478.1 & 213 & -11.6 & -0.4 \\
$\mathrm{H}$ & 180 & 155.7 & 168.3 & 370 & -13.5 & -6.5 \\
$\mathrm{He}$ & 400 & 372.8 & 416.9 & 789 & -6.8 & 4.2 \\
$\mathrm{He}$ & 207.8 & 197.9 & 207.9 & 846 & -4.8 & 0.0 \\
$\mathrm{He}$ & 113.5 & 61.6 & 105.9 & 933 & -45.7 & -6.7 \\
$\mathrm{H}$ & 28 & 25.4 & 27.4 & 1049 & -9.3 & -2.1 \\
$\mathrm{C}$ & 180 & 105.2 & 174.3 & 4415 & -41.6 & -3.2 \\
$\mathrm{O}$ & 430 & 200.4 & 418.9 & 4500 & -53.4 & -2.6 \\
$\mathrm{Ne}$ & 498.4 & 225.6 & 543.9 & 4546 & -54.7 & 9.1 \\
\hline \hline
\end{tabular}




\section{ACKNOWLEDGMENTS}

This work was done with support from Wyle Bioastronautics and Human Health and Performance Contract programs. We would like to acknowledge our colleagues from Tandem Van de Graaff facility at Brookhaven National Laboratory for all their help with collecting the data necessary for this publication.

${ }^{1}$ A. A. Bahadori, E. J. Semones, R. Gaza, M. Kroupa, R. R. Rios, N. N. Stoffle, T. Campbell-Ricketts, L. S. Pinsky, and D. Turecek, "Battery-operated independent radiation detector data report from exploration flight test 1,"NASA/TP-2015-218575, 2015, http://ston.jsc.nasa.gov/ collections/TRS/_techrep/TP-2015-218575.pdf.

${ }^{2}$ M. Kroupa et al., "A semiconductor radiation imaging pixel detector for space radiation dosimetry," Life Sci. Space Res. 6, 69-78 (2015).

${ }^{3}$ L. S. Pinsky, J. Idarraga-Munoz, M. Kroupa, H. M. Son, N. N. Stoffle, E. A. Semones, A. A. Bahadori, D. Turecek, S. Pospísil, J. Jakubek, Z. Vykydal, H. Kitamura, and Y. Uchihori, "Medipix in space on-board the ISS," J. Radiat. Res. 55, i62-i63 (2014).

${ }^{4}$ N. Stoffle, L. Pinsky, M. Kroupa, S. Hoang, J. Idarraga, C. Amberboy, R. Rios, J. Hauss, J. Keller, A. Bahadori, E. Semones, D. Turecek, J. Jakubek, Z. Vyky-dal, and S. Pospisil, "Timepix-based radiation environment monitor measurements aboard the international space station," Nucl. Instrum. Methods Phys. Res., Sect. A 782, 143-148 (2015).

${ }^{5}$ S. M. Hoang, "A pattern recognition approach to learning tracks of heavyion particles in timepix detectors," Ph.D. thesis, University of Houston, Houston, TX, USA, 2013.

${ }^{6}$ S. Hoang, L. Pinsky, R. Vilalta, and J. Jakubek, "LET estimation of heavy ion particles based on timepix-based Si detector," J. Phys.: Conf. Ser. 396, 022023 (2012).

${ }^{7}$ T. Holy, E. Heijne, J. Jakubek, S. Pospisil, J. Uher, and Z. Vykydal, "Pattern recognition of tracks induced by individual quanta of ionizing radiation in medipix 2 silicon detector,” Nucl. Instrum. Methods Phys. Res., Sect. A 591, 287-290 (2008).
${ }^{8}$ X. Llopart, R. Ballabriga, M. Campbell, L. Tlustos, and W. Wong, "Timepix, a 65k programmable pixel readout chip for arrival time, energy and/or photon counting measurements," Nucl. Instrum. Methods Phys. Res., Sect. A 581(1), 485-494 (2007).

${ }^{9}$ M. Campbell, "10 years of the medipix 2 collaboration," Nucl. Instrum. Methods Phys. Res., Sect. A 633, S1-S10 (2010).

${ }^{10} \mathrm{~F}$. Krummenacher, "Pixel detectors with local intelligence: An IC designer point of view," Nucl. Instrum. Methods Phys. Res., Sect. A 305, 527-532 (1991).

${ }^{11}$ J. Jakubek, "Precise energy calibration of pixel detector working in timeover-threshold mode," Nucl. Instrum. Methods Phys. Res., Sect. A 633, S262-S266 (2011).

${ }^{12} \mathrm{M}$. Kroupa et al., "Energy resolution and power consumption of timepix detector for different detector settings and saturation of front-end electronics," J. Instrum. 9(05), C05008 (2014).

${ }^{13}$ J. Jakůbek, "Energy sensitive X-ray radiography and charge sharing effect in pixelated detector," Nucl. Instrum. Methods Phys. Res., Sect. A 607(1), 192-195 (2009).

${ }^{14}$ J. Bouchami, A. Gutierrez, A. Houdayer, J. Idarraga, J. Jakủbek, C. Lebel, C. Leroy, J. Martin, M. Platkevič, and S. Pospísill, "Study of the charge sharing in silicon pixel detector with heavy ionizing particles interacting with a medipix 2 device," Nucl. Instrum. Methods Phys. Res., Sect. A 607(1), 196-198 (2009)

${ }^{15}$ M. Campbell, E. Heijne, T. Holy, J. Idarraga, J. Jakubek, C. Lebel, C. Leroy, X. Llopart, S. Pospisil, L. Tlustos, and Z. Vykydal, "Study of the charge sharing in a silicon pixel detector by means of $\alpha$-particles interacting with a medipix 2 device," Nucl. Instrum. Methods Phys. Res., Sect. A 591, 38-41 (2008).

${ }^{16}$ M. Kroupa, J. Jakůbek, and F. Krejčí, "Charge collection characterization with semiconductor pixel detector timepix," in IEEE NSS/MIC/RTSD Conference Proceedings (IEEE, 2008), pp. R12-R37.

${ }^{17}$ T. T. Boehlen, F. Cerutti, M. P. W. Chin, A. Fasso, A. Ferrari, P. G. Ortega, A. Mairani, P. R. Sala, G. Smirnov, and V. Vlachoudis, "The FLUKA code: Developments and challenges for high energy and medical applications," Nucl. Data Sheets 120, 211-214 (2014).

${ }^{18}$ A. Fasso, A. Ferrari, J. Ranft, and P. Sala, "FLUKA: A multi-particle transport code," CERN-2005-10, INFN/TC-05/11, SLAC-R-773, 2005. 